\title{
Backstepping Control for the Schrödinger Equation with an Arbitrary Potential in a Confined Space
}

\author{
Pedro Franco $\mathbb{D},{ }^{1}$ A. Schaum $\mathbb{D},{ }^{2}$ and Roberto Bernal Jaquez $\mathbb{D}^{3}$ \\ ${ }^{1}$ Posgrado en Ciencias Naturales e Ingeniería, Universidad Autónoma Metropolitana-Cuajimalpa, Mexico City, Mexico \\ ${ }^{2}$ Chair of Automatic Control, Kiel University, Kiel, Germany \\ ${ }^{3}$ Departamento de Matemáticas Aplicadas y Sistemas, Universidad Autónoma Metropolitana-Cuajimalpa, Mexico City, Mexico
}

Correspondence should be addressed to A. Schaum; alsc@tf.uni-kiel.de

Received 28 February 2018; Accepted 20 June 2018; Published 19 July 2018

Academic Editor: Xiaoliang Jin

Copyright (C) 2018 Pedro Franco et al. This is an open access article distributed under the Creative Commons Attribution License, which permits unrestricted use, distribution, and reproduction in any medium, provided the original work is properly cited.

\begin{abstract}
In this work the control design problem for the Schrödinger equation with an arbitrary potential is addressed. In particular a controller is designed which (i) for a space-dependent potential steers the state probability density function to a prescribed solution and (ii) for a space and state-dependent potential exponentially stabilizes the zero solution. The problem is addressed using a backstepping controller that steers to zero the deviation between the initial probability wave function and the target probability wave function. The exponential convergence property is rigorously established and the convergence behavior is illustrated using numerical simulations for the Morse and the Pöschl-Teller potentials as well as the semilinear Schrödinger equation with cubic potential.
\end{abstract}

\section{Introduction}

The problem of quantum control is an important problem in today's quantum technology with relevant applications in quantum information systems, molecular chemistry, and atomic physics, among others [1-3]. Even though control theory of classical mechanical systems has been extensively developed and applied in recent years, its application to quantum mechanical systems bears some additional hurdles due to particularities of the quantum world which in particular impose restrictions on the controllability of these systems [4-6]. In spite of the progress made in this field, still more research and understanding of the underlying quantum phenomena are necessary in order to fully achieve control in the quantum mechanical realm [7-9]. A particularly interesting approach to the boundary control of Partial Differential Equations (PDEs) in general and Schrödinger equations in particular is the backstepping method [10, 11], yielding exponential stabilization of the $L^{2}$-norm in the case of a potential-free Schrödinger equation. This method is used in a variety of engineering problems $[12,13]$ and has been recently extended to the case of regulator design for a Schrödinger equation with a general purpose space-dependent potential and subject to distributed and boundary perturbations [14]. It is an interesting question, which to the authors knowledge still remains open, how these approaches perform for particular potentials of interest, like the Morse potential [15-17] or the Pöschl-Teller potential [18-21]. Furthermore, the aforementioned results are restricted to potentials which are not a function of the state, i.e., to the case of linear Schrödinger equations. Nevertheless, important application examples require the consideration of state-dependent potentials, e.g., to describe the evolution of solitons in optical fibers $[22,23]$ or the description of deep water waves [24]. This leads to a semilinear Schrödinger equation. As it is well-known, the application of the backstepping approach to semilinear systems is only resolved for particular cases [25-29]. Accordingly, the problem of controlling a semilinear Schrödinger equation is still an open task.

In this work, we consider the problem of steering a Probability Density Function (PDF) into a target PDF. It should be noted that the use of the PDF control perspective has not been widely explored [30]. In particular, for space-dependent potentials convergence to an arbitrary desired solution is achieved, and for state-dependent potentials, i.e., the case of a semilinear Schrödinger equation, the zero solution is 
exponentially stabilized. This task is resolved by using boundary actuation following the backstepping method [10]. In contrast to transforming the complete Schrödinger equation into a target PDE, the system is viewed as an interconnection of a linear dynamic with a nonlinear static subsystem, just as in the framework of absolute stability analysis [31,32], and the backstepping transformation is performed only for the linear subsystem.

The paper is organized as follows. In Section 2, the problem is stated from the perspective of PDF control and the initial and target systems are given. In Section 3, the boundary control is designed and the main results of this work are given. In Section 4, to illustrate our findings, we set the control for three significant physical systems in order to show the validity of the method developed in this paper. In Section 4.1, we control a Schrödinger system with a Morse potential, and a system with a Pöschl-Teller potential is examined in Section 4.2. In Section 4.3 the exponential stabilization of the solution for the semilinear Schrödinger equation is considered. Conclusions are given in Section 5.

\section{Problem Formulation}

Consider the Schrödinger equation with a nonlinear potential $V$ and boundary control

$$
\begin{aligned}
i \partial_{t} \psi(x, t) & =-\partial_{x}^{2} \psi(x, t)+V(x, \psi(x, t)) \psi(x, t), \\
\psi(x, 0) & =\psi_{0}(x) \\
\partial_{x} \psi(0, t) & =0 \\
\psi(1, t) & =u(t)
\end{aligned}
$$

with the restriction

$$
\int_{0}^{1} \psi(x, t) \overline{\psi(x, t)} d x=1, \quad \forall t \geq 0 .
$$

In (1a), (1b), (1c), and (1d), $x \in[0,1]$ is the spatial coordinate describing the position of a confined particle within a bounded space or cavity, $t$ is the time, $\psi(x, t) \epsilon$ $L^{2}([0,1] \times \mathbb{R}) \longrightarrow \mathbb{C}$ is the complex-valued wave function that gives the quantum state of the system and $\overline{\psi(x, t)}$ is its conjugate complex counterpart, $V(x, \psi(x, t))$ is the real-valued nonlinear potential function determining the movement of the particle within the cavity, and $u(t) \in \mathscr{C}^{\infty}\left(\mathbb{R}_{+}\right)$is the control input. The function $V$ is continuously differentiable with respect to both arguments.

Equation (1d) states that the system is confined into $x \in$ $[0,1]$ and that the probability of finding the particle within the cavity is conserved for all times. Stated in terms of the PDF

$$
\rho(x, t)=\psi(x, t) \overline{\psi(x, t)}
$$

this means that

$$
\int_{0}^{1} \rho(x, t) d x=1, \quad \forall t \geq 0 .
$$

The problems addressed in this paper consist in the design of a control law $u=\omega(t, \psi)$ such that the wave function $\psi(x, t)$ converges exponentially in the $L^{2}$-norm to a target wave function $\psi_{d}(x, t)$, i.e., that there exist constants $M \geq$ $1, \kappa>0$ such that, for all $\mathrm{t} \geq 0$,

$$
\begin{aligned}
\left\|\psi-\psi_{d}\right\| & =\left(\int_{0}^{1}\left|\psi(x, t)-\psi_{d}(x, t)\right|^{2} d x\right)^{1 / 2} \\
& \leq M\left\|\psi_{0}-\psi_{d 0}\right\| e^{-\kappa t}
\end{aligned}
$$

where $\psi_{0}(x)=\psi(x, 0), \psi_{d 0}(x)=\psi_{d}(x, 0)$ denote the initial profiles of $\psi(x, t)$ and $\psi_{d}(x, t)$, respectively, and the target wave function $\psi_{d}(x, t)$ is a solution of the same Schrödinger equation (1a), (1b), (1c), and (1d) with the control input $u(t)=$ $u_{d}(t)$. Two different cases are considered:

(I) For the case of a space-dependent potential $V(x)$ an arbitrary target system can be considered with the state $\psi_{d}(x, t)$ associated with a quantum system with the same potential but different boundary and initial conditions $\psi_{d}(1, t)=u_{d}(t), \partial_{x} \psi_{d}(0, t)=0$, and $\psi_{d}(x, 0)=\psi_{d 0}$.

(II) For the case of a space and state-dependent potential $V(x, \psi(x, t))$ the target state is given by the zero solution $\psi_{d}(x, t)=0 \forall x \in[0,1], t \geq 0$, which corresponds to the solution of (1a), (1b), (1c), and (1d) with initial condition $\psi_{d 0}(x)=0, \forall x \in[0,1]$, and the control input $u_{d}(t)=0, \forall t \geq 0$.

Introducing the deviation variables

$$
\begin{aligned}
\widetilde{\psi}(x, t) & =\psi(x, t)-\psi_{d}(x, t), \\
\tilde{u}(t) & =u(t)-u_{d}(t)
\end{aligned}
$$

where in case (I) $\psi_{d}(x, t) \neq 0$ and in case (II) $\psi_{d}(x, t)=$ $0, u_{d}(t)=0$, it can easily be verified that the associated Schrödinger equations for $\widetilde{\psi}(x, t)$ have the form

$$
\begin{aligned}
i \partial_{t} \widetilde{\psi}(x, t) & =-\partial_{x}^{2} \widetilde{\psi}(x, t)+V(x, \widetilde{\psi}(x, t)) \widetilde{\psi}(x, t), \\
\widetilde{\psi}(x, 0) & =\widetilde{\psi}_{0}(x) \\
\partial_{x} \widetilde{\psi}(0, t) & =0 \\
\widetilde{\psi}(1, t) & =\widetilde{u}(t) .
\end{aligned}
$$

The associated error PDF is given by

$$
\tilde{\rho}(x, t)=\widetilde{\psi}(x, t) \overline{\widetilde{\psi}(x, t)}=\left|\psi(x, t)-\psi_{d}(x, t)\right|^{2} \geq 0
$$

together with the probability of a nonzero estimation error

$$
P(t)=\int_{0}^{1} \tilde{\rho}(x, t) d x
$$

In terms of the PDF $\tilde{\rho}(x, t)$ and the probability $P(t)$ the convergence requirement (4) reads

$$
P(t) \leq M e^{-\kappa t} P(0), \quad P(0)=\int_{0}^{1} \tilde{\rho}(x, 0) d x
$$

and corresponds to the convergence in the $L^{1}$-norm of $\tilde{\rho}$ to zero. 


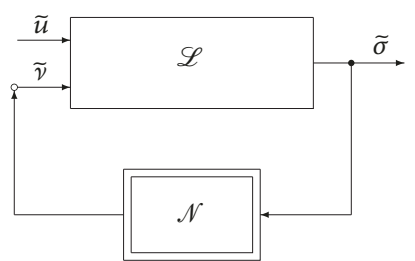

FIGURE 1: Interconnection scheme (10a), (10b), (10c), (10d), and (10e) with $\mathscr{L}$ given by (10a)-(10d) and $\mathcal{N}$ given by (10e).

\section{Control Design}

For the purpose of control design, the dynamics (6a), (6b), and $(6 \mathrm{c})$ are interpreted as an interconnection of a linear dynamical subsystem $\mathscr{L}$ given by (10a)-(10d) with distributed input $\widetilde{\nu}(x, t)$ and output $\widetilde{\sigma}(\mathrm{x}, t)$, and a nonlinear static subsystem $\mathcal{N}$ given by (10e) is defined as follows:

$$
\begin{aligned}
i \partial_{t} \widetilde{\psi}(x, t) & =-\partial_{x}^{2} \widetilde{\psi}(x, t)+\widetilde{v}(x, t), \\
\widetilde{\psi}(x, 0) & =\widetilde{\psi}_{0}(x) \\
\partial_{x} \widetilde{\psi}(0, t) & =0 \\
\widetilde{\psi}(1, t) & =\widetilde{u}(t) \\
\widetilde{\sigma}(x, t) & =\widetilde{\psi}(x, t) \\
\widetilde{\nu}(x, t) & =V(x, \widetilde{\sigma}(x, t)) \widetilde{\sigma}(x, t) .
\end{aligned}
$$

Note that this kind of interpretation of semilinear (or nonlinear) systems in terms of interconnected subsystems is standard in absolute stability theory $[31,32]$ and corresponds to the nonlinear feedback loop sketched in Figure 1.

Accordingly, the main idea behind the approach in the present paper consists in designing a feedback control for the linear part in such a way that the semilinear system given by the two-subsystem interconnection has an exponentially stable zero solution.

3.1. Open-Loop Dynamics. Before addressing the control design problem, the stability properties of the open-loop dynamics are analyzed. The following result establishes the instability of $\widetilde{\rho}(x, t)=0$ and will further be very useful in the subsequent developments.

Lemma 1. For $\widetilde{u}(t)=0$, the probability that the wave function $\psi(x, t)$ does not converge to the desired wave function $\psi_{d}(x, t)$ is conserved over time; i.e.,

$$
\begin{aligned}
\forall t & \geq 0: \\
P(t) & =\int_{0}^{1} \widetilde{\rho}(x, t) d x=K, \\
K & =\text { constant } .
\end{aligned}
$$

Accordingly, the solution $\widetilde{\rho}(x, t)=0$ for the error PDF (7) is unstable.
Proof. A direct calculation shows that

$$
\begin{aligned}
& \frac{d P(t)}{d t}=\int_{0}^{1} \partial_{t} \widetilde{\rho}(x, t) d x=\int_{0}^{1}\left[\left(\partial_{t} \widetilde{\psi}(x, t)\right) \overline{\widetilde{\psi}(x, t)}\right. \\
& \left.+\widetilde{\psi}(x, t)\left(\partial_{t} \overline{\widetilde{\psi}(x, t)}\right)\right] d x \\
& =\int_{0}^{1}\left[\left(i \partial_{x}^{2} \widetilde{\psi}(x, t)-i V(x, \widetilde{\psi}(x, t)) \widetilde{\psi}(x, t)\right)\right. \\
& \cdot \overline{\widetilde{\psi}(x, t)}++\widetilde{\psi}(x, t) \\
& \left.\cdot\left(-i \partial_{x}^{2} \overline{\widetilde{\psi}(x, t)}+i \overline{V(x, \widetilde{\psi}(x, t)) \widetilde{\psi}(x, t)}\right)\right] d x .
\end{aligned}
$$

Taking into account the fact that $V(x, \psi(x, t)) \in \mathbb{R}$, it holds that

$$
V(x, \psi(x, t))=\overline{V(x, \psi(x, t))} \in \mathbb{R} .
$$

Thus, it follows that

$$
\begin{aligned}
& \frac{d P(t)}{d t}=i \int_{0}^{1}\left[\partial_{x}^{2} \widetilde{\psi}(x, t) \overline{\widetilde{\psi}(x, t)}\right. \\
& \left.-\widetilde{\psi}(x, t) \partial_{x}^{2} \overline{\widetilde{\psi}(x, t)}\right] d x \\
& \quad+-i \int_{0}^{1}[V(x, \widetilde{\psi}(x, t)) \widetilde{\psi}(x, t) \overline{\widetilde{\psi}(x, t)} \\
& \quad-\widetilde{\psi}(x, t) V(x, \widetilde{\psi}(x, t)) \overline{\widetilde{\psi}(x, t)}] d x .
\end{aligned}
$$

The second term is clearly zero, and integration by parts of the first term and substitution of the boundary conditions (6b), (6c) yield

$$
\begin{array}{rl}
\int_{0}^{1} & i\left[\partial_{x}^{2} \widetilde{\psi}(x, t) \overline{\widetilde{\psi}(x, t)}-\widetilde{\psi}(x, t) \partial_{x}^{2} \overline{\widetilde{\psi}(x, t)}\right] d x \\
& =i\left[\partial_{x} \widetilde{\psi}(x, t) \overline{\widetilde{\psi}(x, t)}-\widetilde{\psi}(x, t) \partial_{x} \overline{\widetilde{\psi}(x, t)}\right]_{0}^{1} \\
& -i \int_{0}^{1}\left[\partial_{x} \widetilde{\psi}(x, t) \partial_{x} \overline{\widetilde{\psi}(x, t)}\right. \\
& \left.-\partial_{x} \widetilde{\psi}(x, t) \partial_{x} \overline{\widetilde{\psi}(x, t)}\right] d x .
\end{array}
$$

The second term is again zero and from the first term it holds that

$$
\frac{d P(t)}{d t}=2 \mathfrak{J}\left(\partial_{x} \widetilde{\psi}(1, t)\right) \widetilde{u}(t),
$$

and for $\widetilde{u}(t)=0$ it results that the probability $P$ is conserved over time, implying that $\tilde{\rho}(x, t)$ does not converge to zero or, equivalently, that $\widetilde{\rho}_{d}(x, t)=0$ is unstable.

Remark 2. From the preceding proof it follows that the conservation property (11) holds independently of the particular shape of the potential $V(x, \psi(x, t))$. 


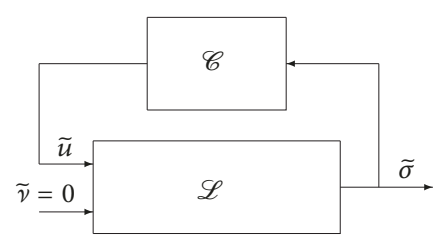

FIGURE 2: Interconnection scheme for the control of the linear Schrödinger system $\mathscr{L}$ with $\widetilde{v}(x, t)=0$ and controller $\mathscr{C}$.

3.2. Control of the Linear Subsystem. Note that the control of the linear subsystem with $\widetilde{v}(x, t)=0$ corresponds to controlling the linear Schrödinger equation without potential, as depicted schematically in Figure 2.

The problem of stabilizing the zero solution of the linear Schrödinger equation has been solved in $[10,11]$. The purpose of this section is to recall the results from $[10,11]$ and to put them in perspective for the analysis of the linear-nonlinear subsystem interconnection (10a), (10b), (10c), (10d), and (10e).

For $\widetilde{\psi}$, a solution of (10a), (10b), (10c), (10d), and (10e) (with $\widetilde{v}(x, t)=0$ ) considers the state transformation $\mathscr{T}$

$$
\tilde{\phi}(x, t)=\mathscr{T} \widetilde{\psi}(x, t)=\widetilde{\psi}(x, t)-\int_{0}^{x} k(x, \xi) \widetilde{\psi}(\xi, t) d \xi
$$

and the target dynamics for $\widetilde{\phi}(x, t)$ given by

$$
\begin{aligned}
i \partial_{t} \tilde{\phi}(x, t) & =-\partial_{x}^{2} \widetilde{\phi}(x, t)-i \gamma \tilde{\phi}(x, t), \\
\tilde{\phi}(x, 0) & =\widetilde{\phi}(x, 0) \\
\partial_{x} \tilde{\phi}(0, t) & =0 \\
\tilde{\phi}(1, t) & =0 .
\end{aligned}
$$

A straightforward calculation (see [10, 11] for details) shows that the dynamics of $\widetilde{\phi}(x, t)$ corresponds to (18a), (18b), and (18c) if and only if the kernel $k(x, \xi)$ of the integral transformation (17) satisfies the PDE

$$
\begin{aligned}
\partial_{x}^{2} k(x, \xi)+\partial_{\xi}^{2} k(x, \xi) & =-i \gamma k(x, \xi) \\
\partial_{\xi} k(x, 0) & =0 \\
k(x, x) & =-\frac{\gamma}{2 i} x .
\end{aligned}
$$

The solution of this PDE is given by (see $[10,11,33])$

$$
k(x, \xi)=i \gamma x \frac{I_{1}\left(\sqrt{-i \gamma\left(x^{2}-\xi^{2}\right)}\right)}{\sqrt{-i \gamma\left(x^{2}-\xi^{2}\right)}},
$$

where $I_{1}(x)$ represents the first-order modified Bessel function of the first kind. From (17) it follows that the control input $\widetilde{u}(t)$ is given by

$$
\begin{aligned}
\tilde{u}(t) & =\int_{0}^{1} k(1, \xi) \widetilde{\psi}(\xi, t) d \xi \\
& =\int_{0}^{1} i \gamma \frac{I_{1}\left(\sqrt{-i \gamma\left(1-\xi^{2}\right)}\right)}{\sqrt{-i \gamma\left(1-\xi^{2}\right)}} \tilde{\psi}(\xi, t) d \xi .
\end{aligned}
$$

For the dynamics (18a), (18b), and (18c), it is known from [11] that the following result holds true.

Lemma 3. The solution $\widetilde{\phi}(x, t)$ of (18a), (18b), and (18c) converges exponentially to zero; i.e., there exist constants $N \geq$ $1, \kappa>0$ such that

$$
\|\widetilde{\phi}(x, t)\| \leq N\|\widetilde{\phi}(x, 0)\| e^{-\kappa t} .
$$

Proof. Consider the $L^{2}$ norm of $\tilde{\phi}$

$$
\|\widetilde{\phi}(x, t)\|=\sqrt{E(t)}, \quad E(t)=\int_{0}^{1} \tilde{\phi}(x, t) \overline{\widetilde{\phi}(x, t)} d x
$$

and recall that it corresponds to the $L^{1}$ norm of the probability density function

$$
\rho_{\tilde{\phi}}(x, t)=\widetilde{\phi}(x, t) \overline{\widetilde{\phi}(x, t)} .
$$

From Lemma 1, it follows that, for $\gamma=0$, the conservation of probability implies that $d E(t) / d t=0$. For $\gamma \neq 0$, it follows from a short calculation that

$$
\begin{aligned}
\frac{d E(t)}{d t} & =\int_{0}^{1}\left[\partial_{t} \widetilde{\phi}(x, t) \overline{\widetilde{\phi}(x, t)}+\widetilde{\phi}(x, t) \partial_{t} \overline{\widetilde{\phi}(x, t)}\right] d x \\
& =-2 \gamma \int_{0}^{1} \widetilde{\phi}(x, t) \overline{\widetilde{\phi}(x, t)} d x=-2 \gamma E(t)
\end{aligned}
$$

implying that

$$
E(t)=E(0) e^{-2 \gamma t}
$$

or equivalently that

$$
\|\widetilde{\phi}(x, t)\|=\|\widetilde{\phi}(x, 0)\| e^{-\gamma t}
$$

meaning that $\widetilde{\phi}(x, t)$ converges exponentially to zero; i.e., inequality (4) holds for $\tilde{\phi}$ with $N=1$ and rate $\kappa=\gamma>0$.

Given the invertibility of the integral transformation (17) (see [11]), it holds that $\tilde{\psi}$ also converges exponentially to zero in the $L^{2}$-norm or, equivalently, $\tilde{\rho}$ converges exponentially to zero in the $L^{1}$-norm. This is summarized in the following corollary.

Lemma 4. Let the control $\tilde{u}(t)$ be chosen according to (21) for some $\gamma>0$. Then, for $\widetilde{\nu}(x, t)=0$, it holds that the deviation state $\widetilde{\psi}(x, t)$ defined in (5) converges exponentially to zero and the probability $P(t)$ defined in (8) satisfies

$$
P(t) \leq M e^{-2 \gamma t} P(0)
$$

for some $M>0$; i.e., (9) is satisfied. 


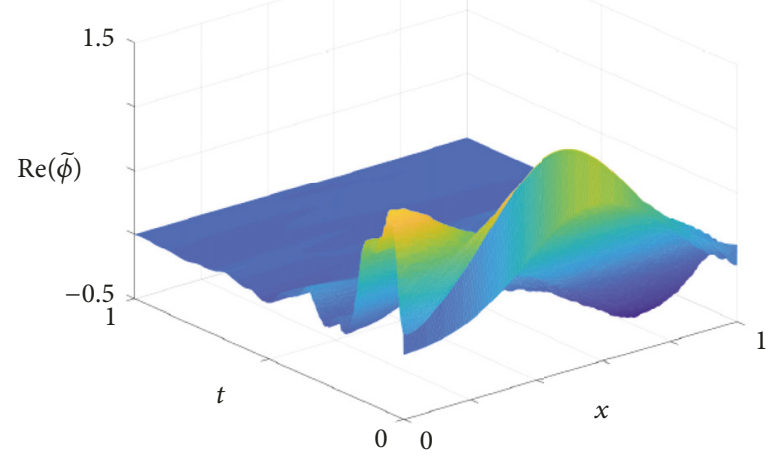

(a) Real part of $\tilde{\phi}$

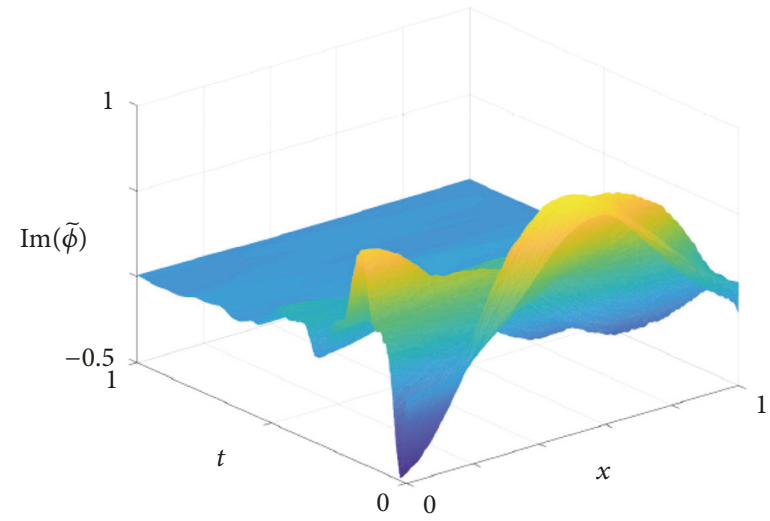

(b) Imaginary part of $\tilde{\phi}$

FIGURE 3: Real and imaginary parts of the solution $\widetilde{\phi}$ of (18a), (18b), and (10c).

Proof. From [11] it is known that the inverse transformation for (17) exists, is unique, and is given by

$$
\begin{aligned}
\widetilde{\psi}(x, t) & =\mathscr{T}^{-1} \tilde{\phi}(x, t) \\
& =\widetilde{\phi}(x, t)+\int_{0}^{x} l(x, \xi) \widetilde{\phi}(\xi, t) d \xi
\end{aligned}
$$

with a bounded kernel $l(x, \xi)$ (see, e.g., [11] for details). Both transformations, $\mathscr{T}$ and its inverse $\mathscr{T}^{-1}$, are linear and bounded operators, which implies that the following inequalities hold:

$$
\begin{aligned}
\|\widetilde{\psi}(x, t)\|^{2} & =\left\|\mathscr{T}^{-1} \tilde{\phi}(x, t)\right\|^{2} \leq\left\|\mathscr{T}^{-1}\right\|_{o p}^{2}\|\widetilde{\phi}(x, t)\|^{2} \\
& \leq N e^{-2 \gamma t}\left\|\mathscr{T}^{-1}\right\|_{o p}^{2}\|\widetilde{\phi}(x, 0)\|^{2} \\
& =N e^{-2 \gamma t}\left\|\mathscr{T}^{-1}\right\|_{o p}^{2}\|\mathscr{T} \tilde{\psi}(x, 0)\|^{2} \\
& \leq N e^{-2 \gamma t}\left\|\mathscr{T}^{-1}\right\|_{o p}^{2}\|\mathscr{T}\|_{o p}^{2}\|\widetilde{\psi}(x, 0)\|^{2}
\end{aligned}
$$

where $\|\cdot\|_{o p}$ denotes the norm of an operator, defined by (see [34])

$$
\|\mathscr{T}\|_{o p}=\sup \{\|\mathscr{T} \psi\|:\|\psi\|=1\}
$$

and in the second inequality, Lemma 3 was used. This implies that

$$
\|\widetilde{\psi}(x, t)\|^{2} \leq M e^{-2 \gamma t}\|\widetilde{\psi}(x, 0)\|^{2}
$$

or, equivalently, that

$$
P(t)=\|\widetilde{\psi}(x, t)\|^{2} \leq M e^{-2 \gamma t} P(0)
$$

with $M=N\left\|\mathscr{T}^{-1}\right\|_{o p}^{2}\|\mathscr{T}\|_{o p}^{2} \geq 1$.

From the preceding lemma the following useful corollary is obtained.
Corollary 5. For $\widetilde{u}(t)$ given by (21) the differential equation

$$
\frac{d P(t)}{d t}=2 \widetilde{u} \Im\left(\partial_{x} \widetilde{\psi}(1, t)\right)
$$

with $\widetilde{\psi}(x, t)$ being the solution of the Schrödinger equation (10a), (10b), (10c), (10d), and (10e) with $\widetilde{v}(x, t)=0$ has a solution $P(t)$ which satisfies (28).

Proof. It follows from (16) and Lemma 4.

To illustrate the functioning of the backstepping controller, in Figure 3 the real and imaginary parts of the solution $\widetilde{\phi}(x, t)$ of $(18 \mathrm{a}),(18 \mathrm{~b})$, and (18c) are shown for the initial condition

$$
\widetilde{\phi}(x, 0)=A e^{-\sigma\left(x-x_{0}\right)^{2} e^{-i x}}
$$

with $A=\sqrt{2}, \sigma=10$, and $x_{0}=0.5$. It can be seen that both real and imaginary parts of $\widetilde{\phi}(x, t)$ converge exponentially to zero, as stated in Lemma 3.

3.2.1. Arbitrary Potential Case. In this subsection, the main result of this work is obtained by proving that, in the presence of an arbitrary $V(x, \psi(x, t))$, the controller (21) stabilizes the origin for the dynamics (6a), (6b), and (6c), which in turn means that $\psi(x, t)$ converges exponentially to $\psi_{d}(x, t)$. In terms of the system-interconnection structure presented in Figure 4, this means that the controller yields absolute stability of the linear subsystem with respect to the nonlinear feedback.

The main result is stated in the next theorem.

Theorem 6. For an arbitrary potential $V(x, \widetilde{\psi}(x, t))$, the error state $\tilde{\psi}(x, t)$ satisfying $(6 a),(6 b)$, and $(6 c)$ converges exponentially to zero if the control $\tilde{u}(t)$ is chosen according to (21), i.e.,

$$
\|\widetilde{\psi}(x, t)\|^{2}=\int_{0}^{1} \tilde{\rho}(x, t) d x \leq M e^{-2 \gamma t}\left\|\widetilde{\psi_{0}}(x)\right\|^{2}
$$

with $M$ given in (28). 


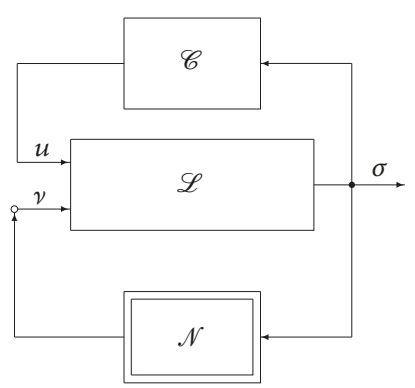

Figure 4: Interconnection scheme (10a), (10b), (10c), (10d), and (10e) with $\mathscr{L}$ given by (10a)-(10d), $\mathscr{N}$ by (10e), and $\mathscr{C}$ by (21).

Proof. From (16) in the proof of Lemma, 1 it follows that

$$
\frac{d P(t)}{d t}=2 \widetilde{u} \Im\left(\partial_{x} \widetilde{\psi}(1, t)\right)
$$

holds for (10a), (10b), (10c), (10d), and (10e) for an arbitrary potential $V(x, \widetilde{\psi}(x, t))$. Thus, the rate of change of the probability does not depend on the potential and, as a consequence of Corollary 5, by applying the control (21) with $\gamma>0$ it follows that

$$
P(t) \leq M e^{-2 \gamma t} P(0),
$$

with $M$ given in (28). This implies that $\widetilde{\psi}(x, t)$ converges exponentially to zero.

\section{Application Examples}

In this section, the stabilization of the Schrödinger equation is studied for three different potentials. In the first case, the Schrödinger equation with a Morse potential [15] is considered. This potential has been extensively used to model the interatomic potential energy of the diatomic molecule in the study of anharmonic vibrations [15-17]. Coupled Morse potentials have been used to model bond interactions and describe the vibrational states of complex molecules [20]. In Section 4.2 the control of the Schrödinger equation with a Pöschl-Teller potential [18] is studied. The study of this potential is important in the description of out of plane molecular vibrations [20], diatomic vibrations [21], and atomic and neutron scattering modeling, among others [19]. Both potentials are shown in Figure 5.

In Section 4.3 a cubic state-dependent potential is considered which corresponds to the study of solitons in optical fibers $[22,23]$ or the description of deep water waves [24].

For the associated stabilization tasks, the control law (21) is applied to the initial system (1a), (1b), (1c), and (1d) by setting

$$
u(t)=u_{d}(t)+\widetilde{u}(t)
$$

with

$$
\begin{gathered}
u_{d}(t)=2 \sin (2 t), \\
\gamma=10 .
\end{gathered}
$$

The resulting Schrödinger equations are solved using the time-splitting finite difference (TSFD) method [35].
4.1. Morse Potential. Consider the Schrödinger equation with a Morse potential

$$
V(x)=D_{e}\left(1-e^{-a\left(x-x_{e}\right)}\right)
$$

with depth $D_{e}$ and width $a$, and an initial condition as defined in (35) (with $A=\sqrt[4]{100 / \pi}, \sigma=50$ and $x_{0}=0.3$ ), i.e., a Gaussian function modeling a wave packet.

The target wave function is defined as the solution of the same equation with a translated initial condition $\psi_{0 d}$ with respect to the initial system $\psi_{0}\left(x_{0}=0.7\right)$ and boundary condition $u_{d}(t)=2 \sin (2 t)$

Making the state transformation (17) on the error variable (5) with $k(x, \xi)$ given in (20) and choosing the control $\tilde{u}(t)$ according to $(21), \widetilde{\psi}(x, t)$ satisfies $(36)$; i.e., $\|\widetilde{\psi}(x, t)\| \longrightarrow 0$. This, in turn, means that $\left\|\psi(x, t)-\psi_{d}(x, t)\right\| \longrightarrow 0$.

Figure 6 shows the initial system, the target system, and the error system, along with the controlled system for $\psi$ which can be seen to be converging to $\psi_{d}$.

4.2. Pöschl-Teller Potential. We consider now the stabilization of the Schrödinger equation (1a), (1b), (1c), and (1d) with a Pöschl-Teller potential given by

$$
V(x)=-\frac{\lambda(\lambda+1)}{2} \operatorname{sech}^{2}\left(a\left(x-x_{e}\right)\right)
$$

with the same initial and boundary conditions used in the example of the Morse potential. Using the same target wave function as in the previous example and error variable (5), we make the state transformation (17) with $k(x, \xi)$ given in (20). Once more, choosing the control $\tilde{u}(t)$ according to (21), $\widetilde{\psi}(x, t)$ satisfies $(36)$; i.e., $\|\widetilde{\psi}(x, t)\| \longrightarrow 0$. This, in turn, means that $\left\|\psi(x, t)-\psi_{d}(x, t)\right\| \longrightarrow 0$ as in our last example. The stabilization for this system is shown in Figure 7.

4.3. Semilinear Schrödinger Equation. In the semilinear Schrödinger equation, the potential is given by

$$
V(x, \psi(x, t))=-|\psi(x, t)|^{2} \psi(x, t)
$$

and the target wave function is the zero solution $\psi_{d}(x, t)=0$. The initial condition chosen for this simulation is a soliton centered in $x=0.2$

$$
\psi_{0}=\sqrt{2} e^{-i(x-.2) / 2} \cosh [20(x-.2)],
$$

and the kernel was chosen equal to the preceding cases. Simulation results are shown in Figure 8.

\section{Conclusions}

In this work a control law for the Schrödinger equation with an arbitrary space- and state-dependent potential has been designed to steer the state evolution to match a target wave function in a bounded domain. For the case of a stateindependent potential the target wave function is an arbitrary solution of the Schrödinger equation for a given initial state and input signal, whereas for the case of a state-dependent 


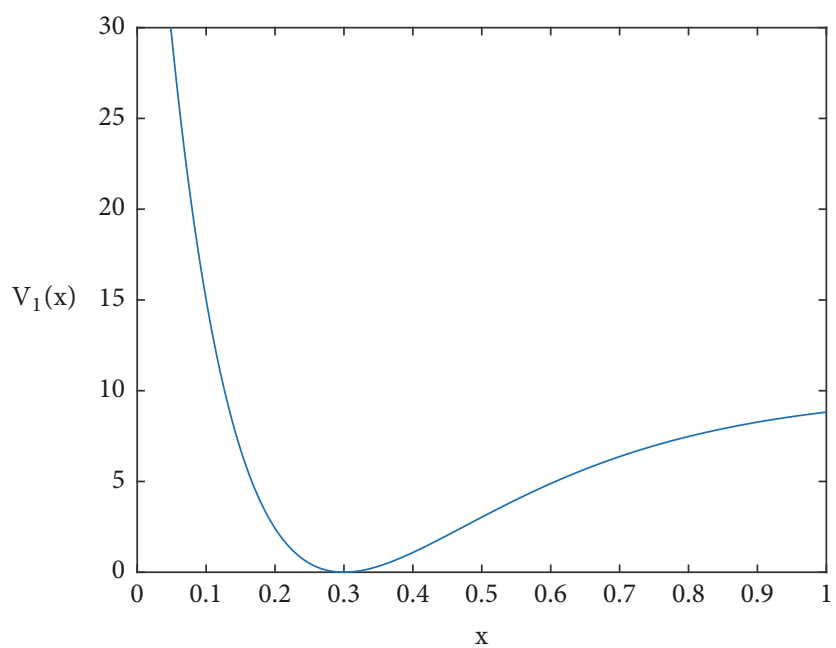

(a) Morse potential with $D_{e}=10, a=4$, and $x_{e}=0.3$

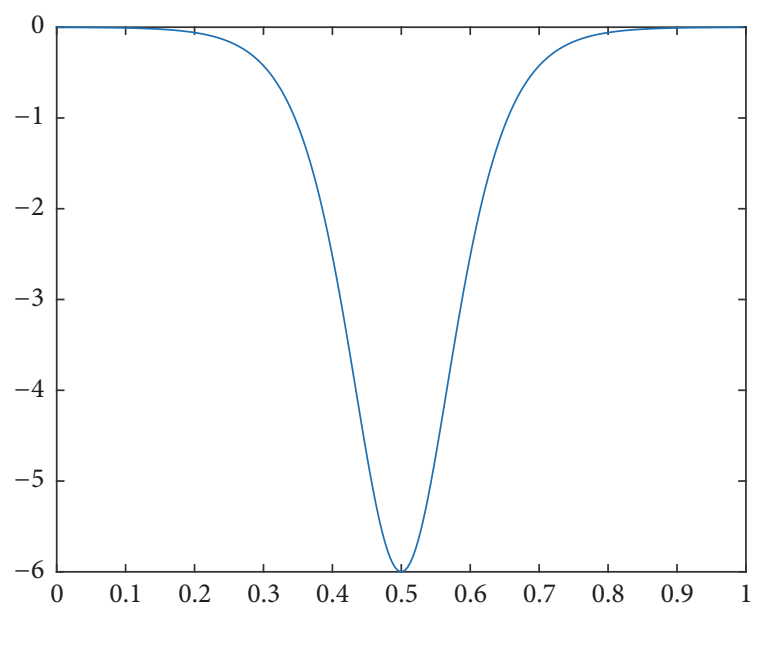

(b) Pöschl-Teller potential with $\lambda=6, a=10$, and $x_{e}=0.5$

Figure 5: Potentials used in examples.

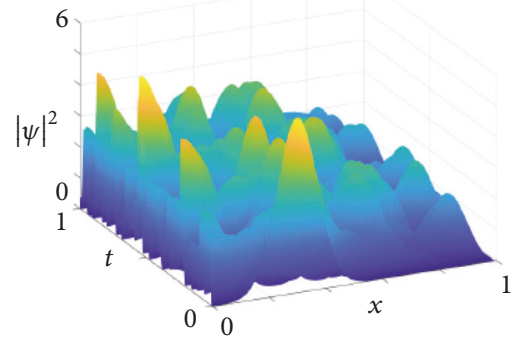

(a)

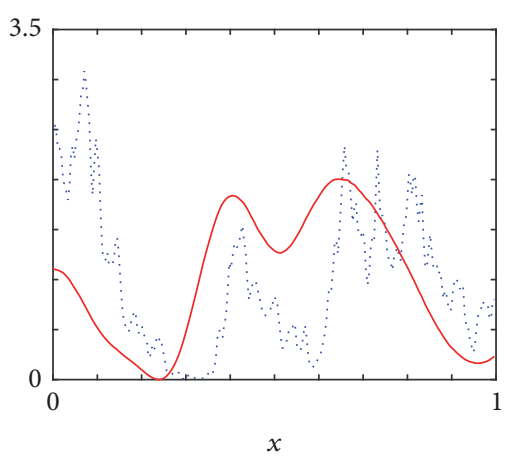

(d)

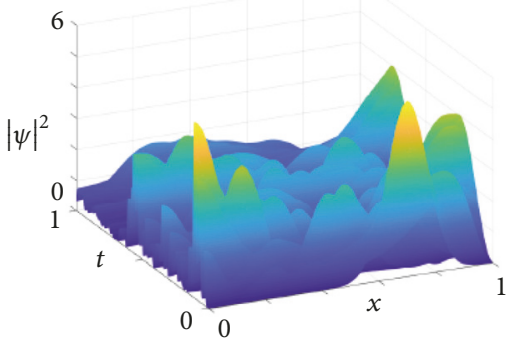

(b)

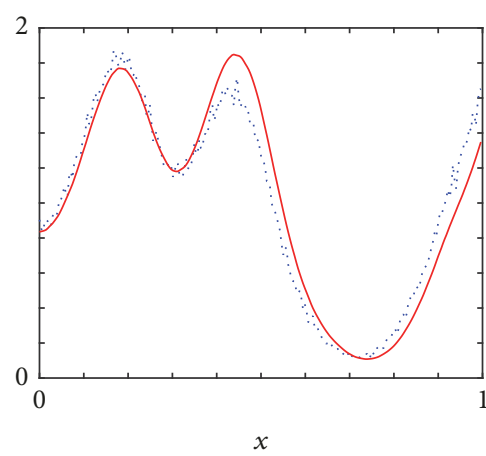

(e)

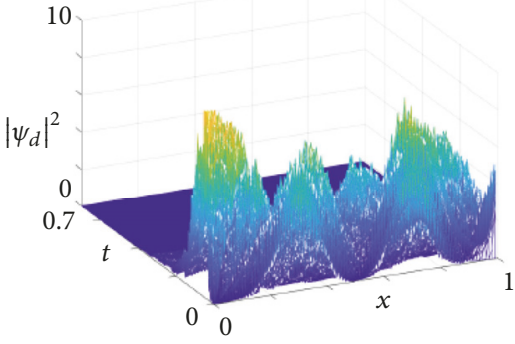

(c)

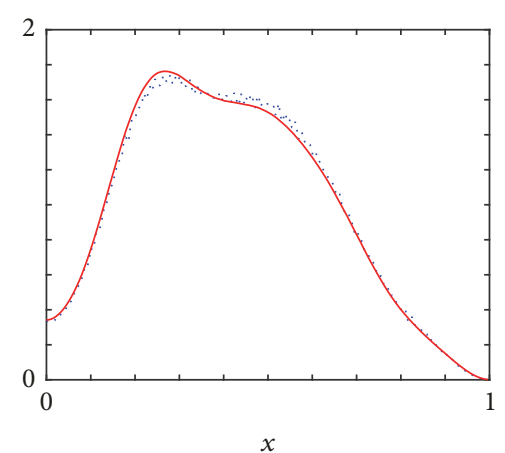

(f)

FIGURE 6: Numerical simulations for the case in which the Morse potential is used. (a) Initial system. (b) Target system. (c) Error system. (d), (e), (f) Comparison between $|\psi|^{2}$ (red) and $\left|\psi_{d}\right|^{2}$ (dotted in blue) at times $\mathrm{t}=0.04, \mathrm{t}=0.6$, and $\mathrm{t}=1$, respectively.

potential the target wave function is given by the zero solution. The control design is carried out by interpreting the semilinear dynamics as a two-subsystem interconnection of a linear dynamic subsystem with a nonlinear static subsystem and following the backstepping design approach for the linear subsystem. The closed-loop stability of the target wave function is rigorously established in terms of the probability of convergence.

In order to illustrate our findings, three different cases of the Schrödinger equation with physically significant potentials have been considered: (i) a Morse potential, (ii) a Pöschl-Teller potential, and (iii) a cubic state-dependent potential. In these examples, numerically solved using the time-splitting finite difference method, it is shown that the designed control makes an initial probability density function to approach and match a target probability density function.

Future research will focus on the question of how to stabilize nonzero target wave functions for the case of the semilinear Schrödinger equation and to include the observer design problem for output-feedback control. 


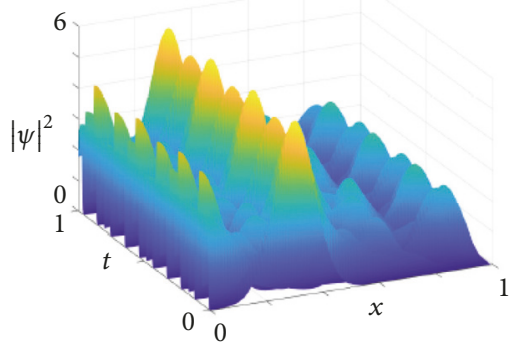

(a)

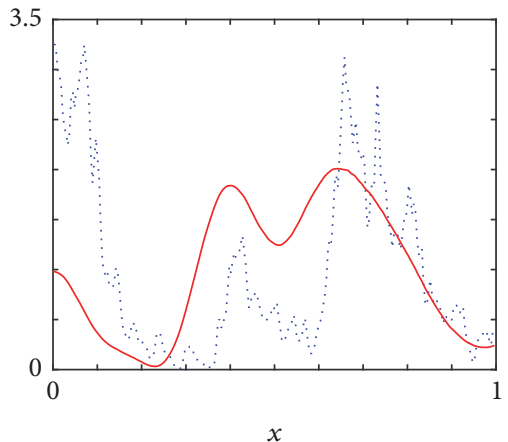

(d)

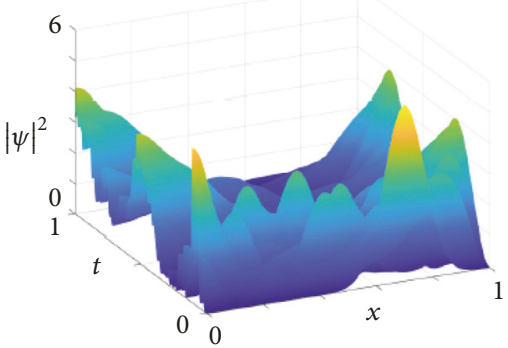

(b)

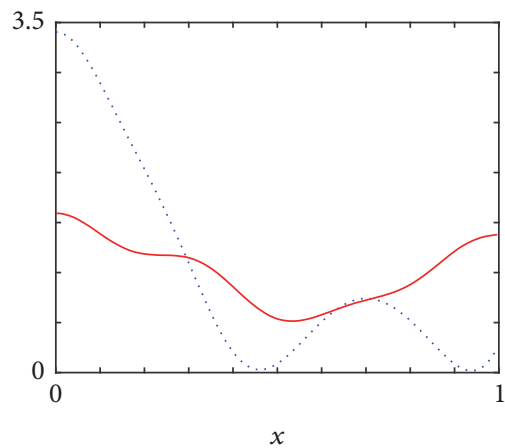

(e)

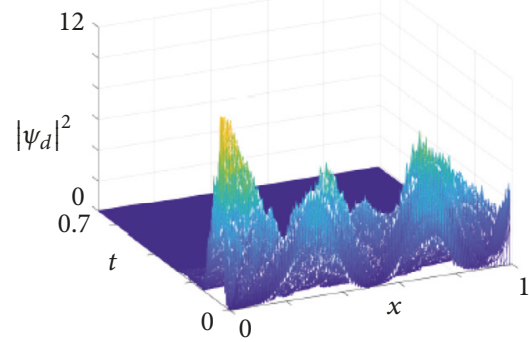

(c)

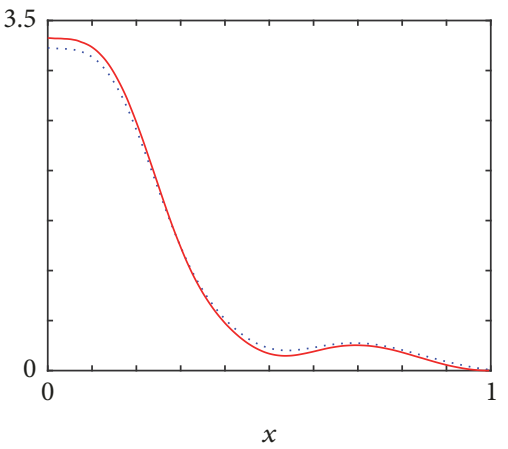

(f)

FIgURE 7: Numerical simulations for the case in which the Pöschl-Teller potential is used. (a) Initial system. (b) Target system. (c) Error system. (d), (e), (f) Comparison between $|\psi|^{2}$ (red) and $\left|\psi_{d}\right|^{2}$ (dotted in blue) at times $\mathrm{t}=0.04, \mathrm{t}=0.6$, and $\mathrm{t}=1$, respectively.

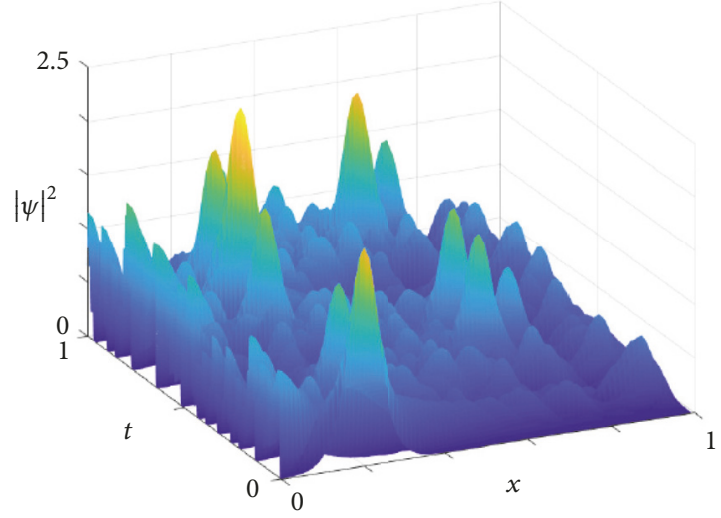

(a) Open-loop system

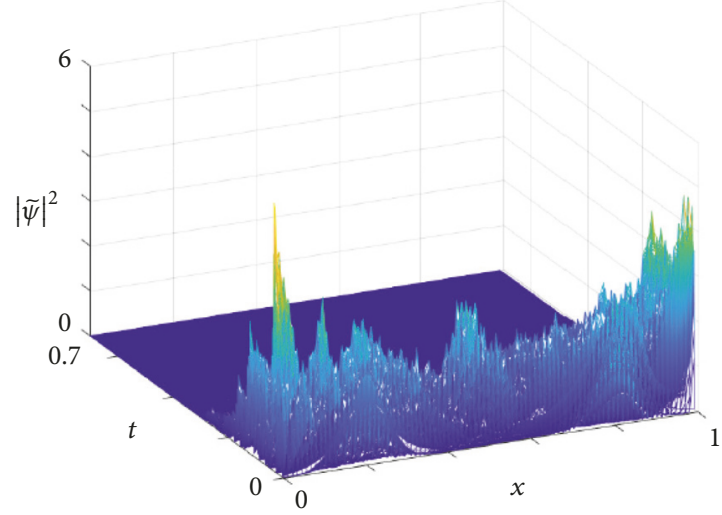

(b) Controlled system

FIGURE 8: Numerical simulations for the case of the semilinear Schrödinger equation with cubic potential (43).

\section{Data Availability}

The data provided in the manuscript is based on numerical simulations using methods which are standard in the field and can thus be reproduced by anyone.

\section{Conflicts of Interest}

The authors declare that they have no conflicts of interest.

\section{Acknowledgments}

P. Franco gratefully acknowledges the financial support of CONACYT Mexico and the Posgrado en Ciencias Naturales e Ingeniería of Universidad Autónoma MetropolitanaCuajimalpa.

\section{References}

[1] H. Rabitz, G. Turinici, and E. Brown, "Control of quantum dynamics: concepts, procedures and future prospects," in Computational Chemistry, Ph. G. Ciarlet, Ed., vol. 10 of Handbook of Numerical Analysis, pp. 833-887, 2003.

[2] H. Rabitz and G. Turinici, "Wavefunction controllability in quantum systems," Journal of Physics A, vol. 36, pp. 2565-2576, 2003.

[3] D. Dong, C. Chen, B. Qi, I. R. Petersen, and F. Nori, "Robust manipulation of superconducting qubits in the presence of fluctuations," Scientific Reports, vol. 5, article 7873, 2015. 
[4] E. Machtyngier, "Exact controllability for the Schrödinger equation," SIAM Journal on Control and Optimization, vol. 51, no. 2, pp. 243-256, 1994.

[5] K.-D. Phung, "Observability and control of Schrödinger equations," SIAM Journal on Control and Optimization, vol. 40, no. 1, pp. 211-230, 2001.

[6] K. Beauchard, "Local controllability of a 1-D Schrödinger equation," Journal de Mathématiques Pures et Appliquées, vol. 84, no. 7, pp. 851-956, 2005.

[7] K. Beauchard and M. Mirrahimi, "Practical stabilization of a quantum particle in a one-dimensional infinite square potential well," SIAM Journal on Control and Optimization, vol. 48, no. 2, pp. 1179-1205, 2009.

[8] W. Kang and E. Fridman, "Sliding mode control of Schrödinger equation-ODE in the presence of unmatched disturbances," Systems \& Control Letters, vol. 98, pp. 65-73, 2016.

[9] C. Chen, D. Dong, B. Qi, I. R. Petersen, and H. Rabitz, "Quantum ensemble classification: a sampling-based learning control approach," IEEE Transactions on Neural Networks and Learning Systems, vol. 28, no. 6, pp. 1345-1359, 2017.

[10] M. Krstic, B. Z. Guo, and A. Smyshlyaev, "Boundary controllers and observers for Schrödinger equation," in Proceedings of the 46th IEEE Conference on Decision and Control, pp. 4149-4154, New Orleans, La, USA, December 2007.

[11] M. Krstic and A. Smyshlyaev, Boundary Control of PDEs: A Course on Backstepping Designs, SIAM Advances in Design and Control, SIAM, Philadelphia, Pa, USA, 2008.

[12] F. Ding, J. Wu, and Y. Wang, "Stabilization of an underactuated surface vessel based on adaptive sliding mode and backstepping control," Mathematical Problems in Engineering, vol. 2013, Article ID 324954, 5 pages, 2013.

[13] X. Huo, M. Huo, and H. R. Karimi, "Attitude stabilization control of a quadrotor UAV by using backstepping approach," Mathematical Problems in Engineering, vol. 2014, Article ID 749803, 9 pages, 2014.

[14] H. C. Zhou and G. Weiss, "Solving the regulator problem for a 1-D Schrödinger equation via backstepping," in Proceedings of the 20th World Congress, pp. 4602-4607, 2017.

[15] P. M. Morse, "Diatomic molecules according to the wave mechanics. II. Vibrational levels," Physical Review A: Atomic, Molecular and Optical Physics, vol. 34, no. 1, pp. 57-64, 1929.

[16] L. de la Peña, Introducción a La Mecánica Cuántica, Universidad Nacional Autónoma de México, Fondo de Cultura Económica, 2nd edition, 1991.

[17] S. Flügge, Practical Quantum Mechanics, Springer, 1998.

[18] G. Pöschl and E. Teller, "Bemerkungen zur Quantenmechanik des anharmonischen Oszillators," Zeitschrift für Physik, vol. 83, no. 3-4, pp. 143-151, 1933.

[19] J. Lekner, "Reflectionless eigenstates of the $\operatorname{sech}^{2}$ potential," American Journal of Physics, vol. 75, no. 12, pp. 1151-1157, 2007.

[20] R. Bernal and R. Lemus, "Algebraic local vibrational spectroscopic description of formaldehyde," Journal of Molecular Spectroscopy, vol. 235, no. 2, pp. 218-234, 2006.

[21] R. Lemus and R. Bernal, "Connection of the vibron model with the modified Pöschl-Teller potential in configuration space," Chemical Physics, vol. 283, no. 3, pp. 401-417, 2002.

[22] S. V. Manakov, "Remarks on the Integrals of the Euler Equations of the n-dimensional Heavy Top," Functional Analysis and Its Applications, vol. 10, no. 4, pp. 93-94, 1976.

[23] C. R. Menyuk, "Application of multiple-length-scale methods to the study of optical fiber transmission," Journal of Engineering Mathematics, vol. 36, no. 1-2, pp. 113-136, 1999.
[24] V. E. Zakharov, "Stability of periodic waves of finite amplitude on the surface of a deep fluid," Journal of Applied Mechanics and Technical Physics, vol. 9, no. 2, pp. 190-194, 1972.

[25] R. Vazquez and M. Krstic, "Control of 1-D parabolic PDEs with Volterra nonlinearities. Part I: design," Automatica, vol. 44, no. 11, pp. 2778-2790, 2008.

[26] R. Vazquez and M. Krstic, "Control of 1D parabolic PDEs with Volterra nonlinearities. Part II: analysis," Automatica, vol. 44, no. 11, pp. 2791-2803, 2008.

[27] J.-M. Coron, R. Vazquez, M. Krstic, and G. Bastin, "Local exponential $\mathrm{H} 2$ stabilization of a $2 \times 2$ quasilinear hyperbolic system using backstepping," SIAM Journal on Control and Optimization, vol. 51, no. 3, pp. 2005-2035, 2013.

[28] A. Hasan, "Backstepping boundary control for semilinear parabolic PDEs," in Proceedings of the 54th IEEE Conference on Decision and Control (CDC '15), pp. 2513-2518, December 2015.

[29] A. Hasan, "Output Feedback Stabilization of Semilinear Parabolic PDEs using backstepping," https://arxiv.org/abs/1612 .03867 .

[30] Y.-F. Xing and J. Wu, "Probability density function control of quantum systems," International Journal of Modern Physics B, vol. 25, no. 17, pp. 2289-2297, 2011.

[31] A. Lur'e and V. N. Postnikov, "On the theory of stability of control systems," Prikladnaya Matematika i Mekhanika, vol. 8, no. 3, pp. 246-248, 1944 (Russian).

[32] M. A. Aizerman and F. R. Gantmacher, Absolute Stability of Regulator Systems, Holden-Day, San Francisco, Calif, USA, 1964.

[33] A. Smyshlyaev and M. Krstic, "Backstepping observers for a class of parabolic PDEs," Systems \& Control Letters, vol. 54, no. 7, pp. 613-625, 2005.

[34] J. B. Conway, A Course in Functional Analysis, Springer Science \& Business Media, Berlin, Germany, 2nd edition, 1994.

[35] X. Antoine, W. Bao, and C. Besse, "Computational methods for the dynamics of the nonlinear Schrödinger/Gross-Pitaevskii equations," Computer Physics Communications, vol. 184, no. 12, pp. 2621-2633, 2013. 


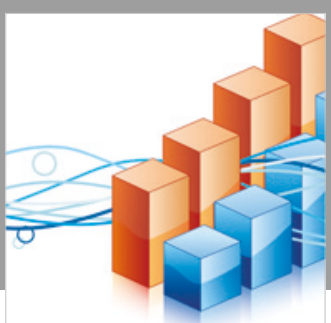

Advances in

Operations Research

\section{-n-m}
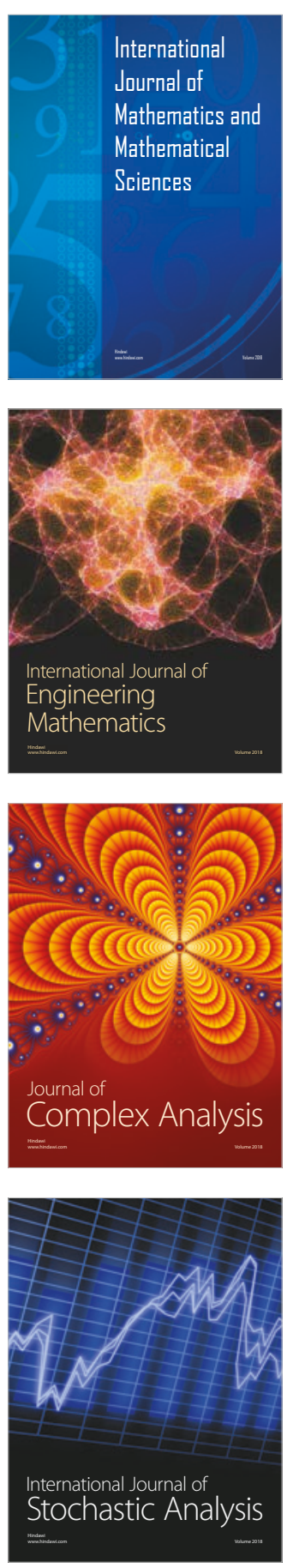
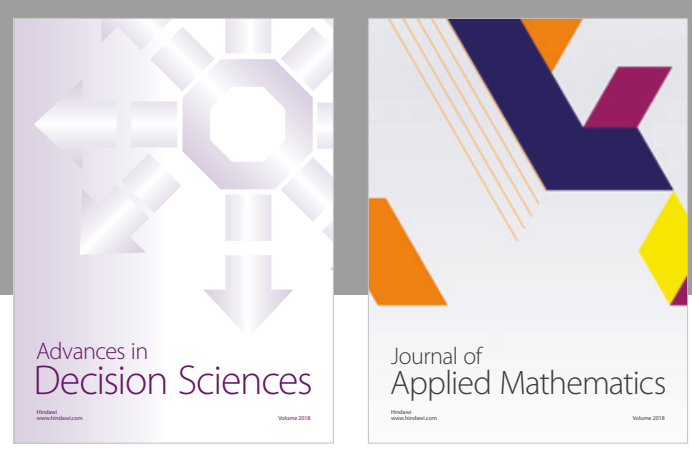

Journal of

Applied Mathematics
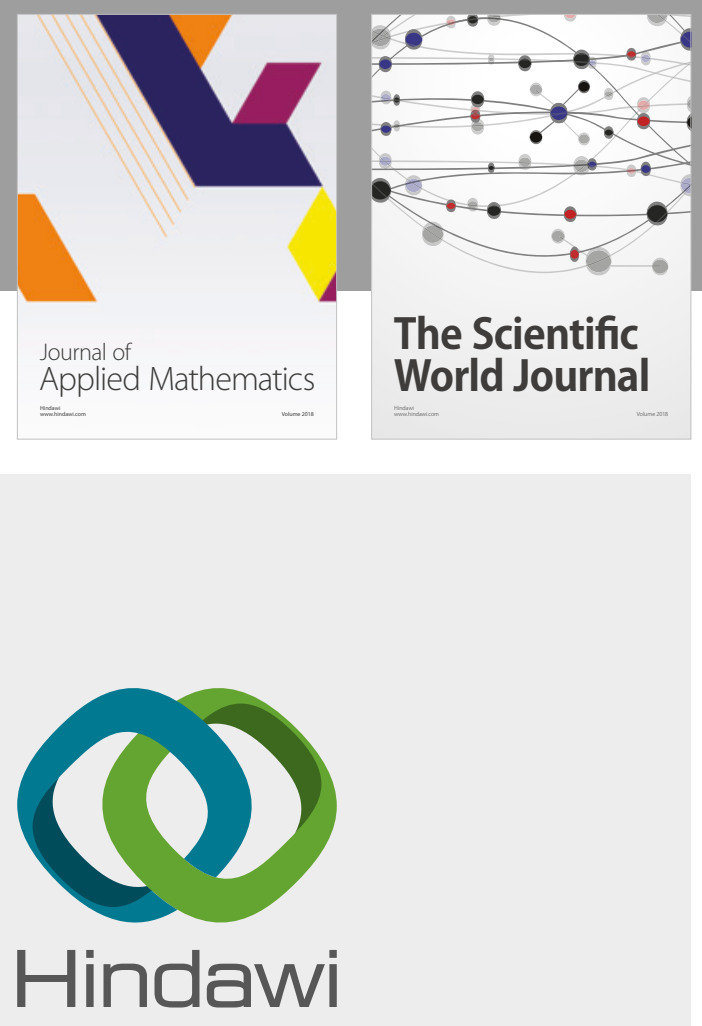

Submit your manuscripts at

www.hindawi.com

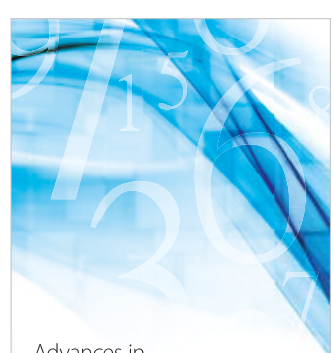

Advances in
Numerical Analysis
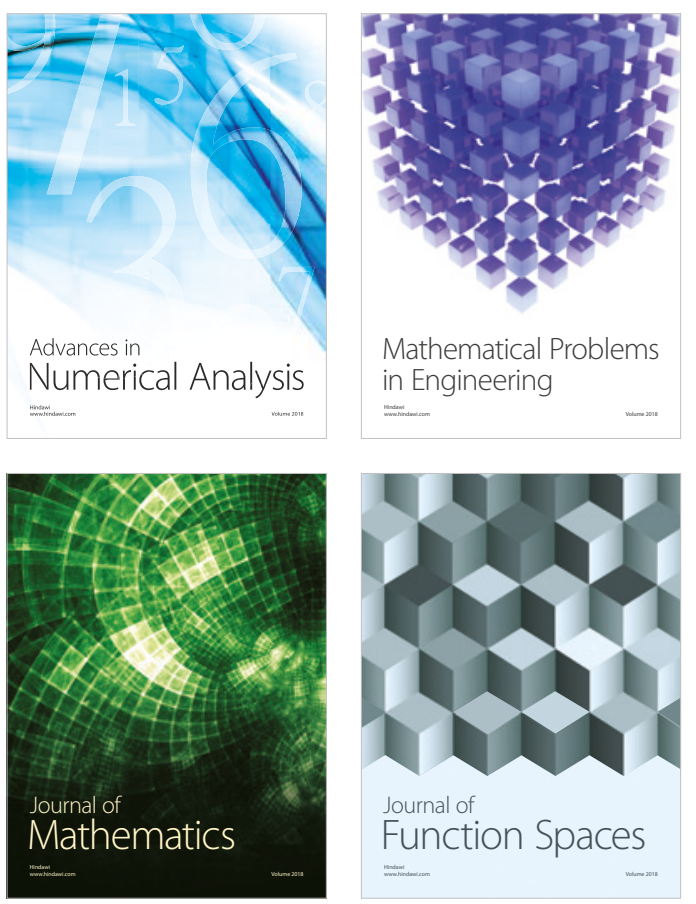

Mathematical Problems in Engineering

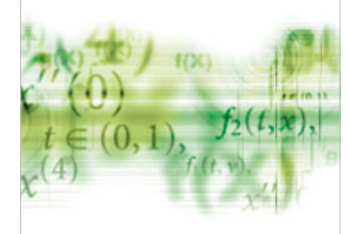

International Journal of

Differential Equations

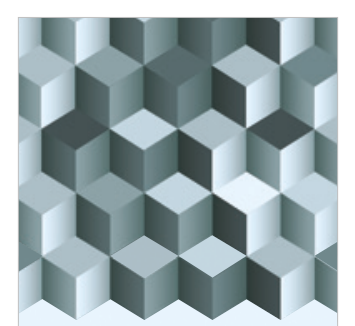

Journal of

Function Spaces

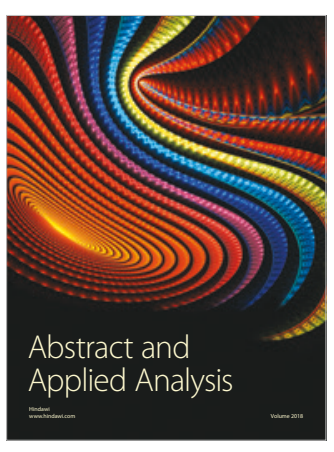

The Scientific

World Journal

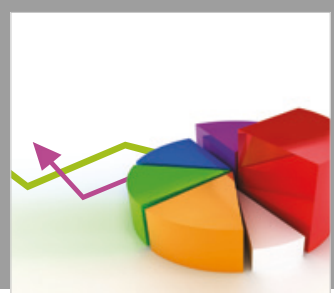

Journal of

Probability and Statistics
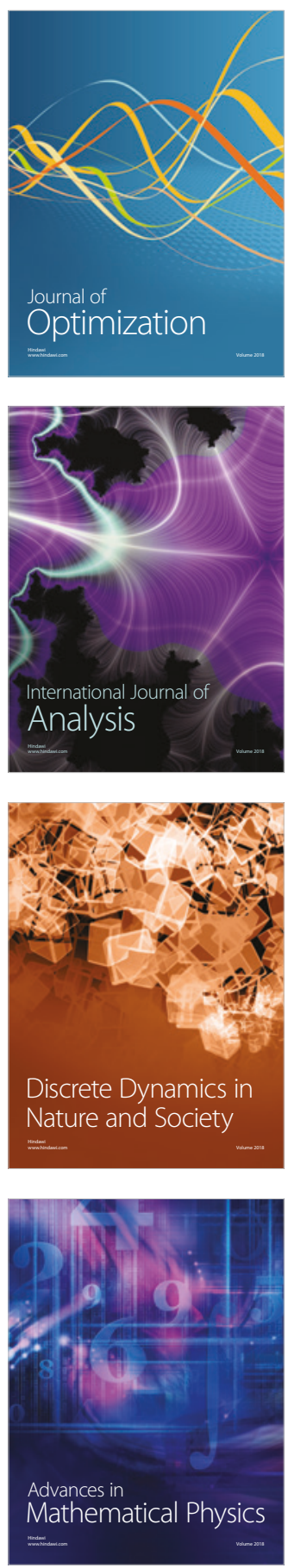